\title{
Determinantes de la cohabitación y el matrimonio en Honduras: población joven en edades de 18 a 29 años, 2005-2012
}

\author{
Juan Carlos Velásquez Espino ${ }^{1}$ \\ Marysabel Zelaya Ochoa ${ }^{2}$
}

\section{RESUMEN}

La cohabitación es un fenómeno que ha estado presente en la sociedad hondureña junto al matrimonio, siendo la primera la forma de unión más practicada por la población, desde edades jóvenes hasta edades adultas. Los porcentajes más altos de cohabitación se presentan en las zonas rurales y son las parejas del nivel educativo con educación primaria quienes más deciden unirse bajo esta modalidad. En la última década este tipo de unión ha ido en aumento.

Se identificaron como los factores que más inciden en este tipo de unión según su significancia estadística: el número de hijos, la edad de la primera relación sexual y la edad de la primera convivencia; pues las personas se casan en edades adultas y cohabitan en edades jóvenes y media adulta.

El presente estudio se plantea identificar las diferencias en las formas de convivencia por edad, lugar de residencia, nivel educativo, religión, número de hijos, edad de la primera unión, edad de la primera relación sexual, religión; asimismo, caracterizar la cohabitación y el matrimonio tomando en cuenta la situación de las mujeres y los hombres en el mercado laboral, ocupación y la tenencia de bienes.

Finalmente, se espera comparar la tendencia hacia el matrimonio o la cohabitación de jóvenes entre los 18 a 29 años en dos momentos distintos: 2005-2006 y 2011-2012. Se toman como unidad de análisis a los jóvenes en edades de 18 a 29 años que se encuentran en unión.

\footnotetext{
1 Beneficiario de una beca especial de la DICYP. Estudiante de la Maestría en Demografía y DesarroIlo, UNAH: Carlos.velas@yahoo.com

2 Profesora universitaria y asesora, Maestría en Demografía y Desarrollo, Facultad de Ciencias Sociales, UNAH: marysabelzo@yahoo.com
} 
Se analizó el caso de hombres y mujeres en edades jóvenes entre 18 y los 29 años de edad, en unión. Para lo cual se utilizó la información de la encuesta nacional de demografía y salud (ENDESA) 2005-2006 y 2011-2012. Para el procesamiento de los datos se hizo uso del programa estadístico SPSS y se aplicó el modelo de regresión logística haciendo uso del programa estadístico Stata.

Palabras clave: cohabitación, matrimonio, unión, jóvenes, familia, edad de la primera unión, edad de la primera relación sexual.

\section{ABSTRACT}

Cohabitation is a phenomenon that has been present in the Honduran society along with marriage, being the first, the most practiced method of union by the population, since their youth to adulthood. The highest rates of cohabitation are shown in rural areas, from couples whose highest educational level is elementary school. This type of union has increased in the last decade.

According to their statistical significance, there were several factors identified in this type of union: the number of children, the age where they had their first intercourse and first cohabitation. People get married in adulthood and cohabit in youth and middle age.

This study attempts to identify differences in forms of cohabitation by age, place of residence, educational level, religion, number of children, which helps characterize cohabitation and marriage considering the situation of working men and women, in relation to their particular occupation and possession of property.

We analyze the case of young men and women between the ages of 18 and 29 years in union. Database given at the National Demographic and Health Survey (DHS) 2005-2006 and 2011-2012, was taken, SPSS statistical program was used to process information. Logistic regression model using the Stata statistical software was applied.

Keywords: cohabitation, marriage, union, youth, family, age at first marriage, age at first sexual relation. 


\section{INTRODUCCIÓN}

La cohabitación ha coexistido en la población hondureña desde tiempos de la colonia y en el transcurso del proceso histórico se ha mantenido por sobre el matrimonio y la unión de hecho. El matrimonio es la unión de dos personas, hombre y mujer, las cuales deciden unir sus vidas y formar una familia, adquiriendo derechos y obligaciones legales por parte de cada uno de los cónyuges.

A nivel mundial el fenómeno de la cohabitación se está incrementando, tal situación también se refleja en Honduras, en donde las parejas cohabitan y comparten costumbres, vidas y familias, pero sin estar casados legalmente.

En la actualidad los factores que conducen a una mujer y a un hombre a convivir, ha cambiado y en este contexto no solo se debe considerar la situación económica, sino también el nivel educativo, el ingreso de las mujeres y los hombres en campo laboral, lo cual puede incidir en el retraso del matrimonio porque lo primero que quieren alcanzar es un lugar dentro del campo laboral, junto a una preparación académica, dejando en segundo plano el tema del matrimonio. Este tipo de coyunturas puede incidir para tomar la decisión de cohabitar, opción que no involucra ningún compromiso legal para la pareja.

La familia tradicional pensada desde el matrimonio legal se va transformando hacia una familia nuclear más moderna, compuesta por padres que viven juntos, pero que no están casados.La evidencia empírica refleja que la cohabitación es mayor que los matrimonios y es lo que se observa en la sociedad hondureña: una prevalencia de la cohabitación por sobre el matrimonio. Con este estudio se pretende presentar el panorama de la cohabitación y el matrimonio en Honduras para el periodo 2011-2012. La investigación se desarrolla en el contexto de Honduras, con jóvenes en las edades de 18 a 29 años que están en cohabitación o matrimonio.

\section{METODOLOGÍA}

La investigación se propuso como un trabajo descriptivo dirigido a responder las interrogantes planteadas en el estudio: ¿Qué diferencias se presentan en la forma de unión según edad, lugar de residencia, nivel educativo, religión, número de hijos, 
edad de la primera unió y edad de la primera relación sexual? ¿Cómo se comportan las diferentes formas de unión según la participación de mujeres y hombres en el mercado laboral? ¿Cuál es la tendencia de la cohabitación y el matrimonio de jóvenes entre 18 a 29 años?

El análisis se centró en las variables demográficas como: estado civil, edad, lugar de residencia, nivel educativo, religión, número de hijos, edad de la primera unión, edad de la primera relación sexual, religión, etnia, mercado laboral, ocupación, tenencia de tierras y tenencia de casa.

Se tomó como fuente de datos la ENDESA 2011-2012, asumiendo como unidad de análisis los hombres y mujeres con estado civil en unión, ya sea casados o en unión libre, en edades entre los 18 y 29 años. Las encuestas de hombres y mujeres son muestras diferentes en las edades de 18 a 29 años que se encontraban en cohabitación o matrimonio. Para el análisis de tendencia se hizo uso de la ENDESA en dos momentos transversales: 2005-2006 y 2011-2012.

Para el procesamiento de los datos se hizo uso del programa estadístico SPSS, los cuales se editaron en Microsoft Excel y para una mejor estética de los cuadros y gráficas se trabajaron con este mismo programa (tabulaciones simples, tablas personalizadas, cruce de variables, filtros de las edades y el estado civil).

Se aplicó el modelo de regresión logística para determinar qué factor incide más en la decisión que toman las personas de cohabitar o casarse, tomando como base la encuesta nacional de demografía y salud de mujeres 2012. Se usaron como referencia los siguientes valores:

1. Variable dependiente: el estado civil, el cual se codificó como cohabita1829= casada y en unión $=1$, distinto de uno $=0$.

2. Se codificaron las variables independientes: religión: católica $=1$, distinto de $1=0$ y evangélica 1 distinto de 1=0;número de uniones: una unión=1, más de una= 0 , tenencia de casa si $=1$ y no $=0$; número de hijos $=$ menos de tres $=1$ y más de tres $=0$, zona de residencia urbano $=1$ y distinto 0 , rural 1 y distinto $=0$; edad de la primera relación sexual: $9-19=1,20$ y más $=0$; edad de la primera convivencia: $7-19$ años $=1,20$ y más $=0$; nivel educativo: se codificó cada uno de los niveles en superior, secundaria, primaria, primaria $=1$ y distinto $=0$. 
Se incluyó todo el país según la ENDESA para 2005-2006³ y para 2011-2012; para la primera se excluyen de la muestra los departamentos de Islas de la Bahía y Gracias a Dios.

\section{ENFOQUES TEÓRICOS DE LA INVESTIGACIÓN}

La unión conyugal se puede explicar desde diferentes perspectivas o enfoques, para la presente investigación se consideran los principios básicos de 4 enfoques:

1. El enfoque de estudio en el curso de vida. En el transcurso de la vida de la personas intervienen diferentes factores que influyen en las decisiones individuales y en el caso de la decisión de unión: el nivel de instrucción, el nivel socioeconómico, la zona de residencia, la religión, el grupo étnico, la edad de la primera relación sexual y la edad de la primera unión; esto significa que la formación de una unión de cohabitación es parte de una secuencia amplia de acontecimientos, enfocando a la familia como un conjunto de carreras individuales mutuamente contingentes, cuya dinámica es precisamente la que da forma a la familia como unidad (Grace y Sweeney, 2014).

2. La teoría del intercambio social. Explica la socialización a la que llegan los hombres y mujeres en unión, los cuales llegan a común acuerdo en las decisiones en conjunto, tomando en cuenta que las personas son interdependientes y que, por lo tanto, necesariamente intercambian cosas (López, 2010).

3. Pesquisa conyugal. Explica que hay diversos factores que influyen en el matrimonio, la persona busca la mejor opción, obteniendo beneficios según el emparejamiento óptimo, adonde los costos y beneficios son influenciados por la educación y el ingreso (Kalmijin, 2011).

4. Segunda transición demográfica. La mayoría de las sociedades actuales tienen las características del aumento de la soltería, retraso de la edad para el matrimonio y remplazo del matrimonio por uniones libres, el cual en ocasiones va precedido de una cohabitación. Las relaciones personales adquieren vital

3 La base de datos de hombres para 2005, fue levantada de forma individual, no se hizo en conjunto con la base de ENDESA de mujeres. 
importancia en la vida de las personas por su deseo de obtener mejoras educativas, salariales y bienes materiales (Von de Kaa, 2002; Lesthaegue, 1994).

\section{ASPECTOS CONCEPTUALES}

La formación de la familia está relacionada directamente con la formación de parejas. En Honduras, las formas de unión que se dan son la cohabitación y el matrimonio.

\section{La cohabitación}

Las características de la cohabitación en Honduras tienen semejanzas con lo que está pasando en Latinoamérica según Grace (2014),adonde se observa la convivencia sin matrimonio, entendiendo el término como la unión de un hombre y una mujer, los cuales mantienen una relación de convivencia sin estar casados legalmente. Las parejas durante el noviazgo toman la decisión de tener o no hijos y las normas y valores que establecerán en el hogar conformado. Si las parejas deciden tener hijos, los van a cuidar, educar, crecer dentro de las normas y valores familiares, prepararlos para entrar a una sociedad que cada día exige más y la capacitación de los jóvenes depende de la que se le facilite desde el seno de su familia.

En la actualidad, las poblaciones ha evolucionado y los cambios son grandes y notorios en las sociedades. La conformación de las parejas, sin lugar a dudas, es una de las transformaciones más vitales que están presentes en la estructuración de la vida familiar; pues los cambios que presentan las poblaciones incluyen modificaciones en la conformación de las parejas. Tal es el caso que señala Quilodran (2003): "El matrimonio ha dejado de ser la norma en los países más desarrollados y en aquellos donde la unión consensual ha sido tradicional, como en el caso de América Latina", así como el mismo explica, en Latinoamérica se avecina y se está viviendo una coexistencia entre la unión libre tradicional y la moderna.

El mundo se encuentra en constantes cambios económicos, políticos, sociales y demográficos; dentro de los últimos se puede observar lo que señala Ron Lesthaeghe (2012) en su conferencia sobre "El boom de la cohabitación en Latinoamérica”; el cual plantea que ya desde el año de 1986 él explica que en las Américas la gente tendría la posibilidad de elegir entre una variedad de modelos de 
familia y la cohabitación fuera del matrimonio sería una de las soluciones más aceptadas.

\section{El matrimonio}

En Honduras, según el Código de la Familia, solamente se reconoce el matrimonio civil celebrado con los requisitos y formalidades, estableciendo la igualdad jurídica entre los contrayentes. El matrimonio reconocido legalmente es el civil, todo ministro de cualquier culto religioso para autorizar el matrimonio religioso debe exigir la certificación de haberse celebrado el matrimonio civil.

La mayoría de edad en Honduras se obtiene al cumplir los 21 años, sin embargo, podrán contraer matrimonio el varón de 18 años y la mujer mayor de 16 años, siempre que se cuente con la autorización del padre y la madre conjuntamente 0 aquel de ellos que ejerza la patria potestad; a falta de los padres, los abuelos maternos o paternos o los adoptantes en caso de ser adoptado (Art. 17, Código de la Familia de Honduras).

La configuración de las familias en la actualidad se torna un tanto diferente de lo que se acostumbra a entender por familia: esta es el núcleo principal de la sociedad (Artículo 16 de la Declaración Universal de los Derechos Humanos, Asamblea General de las Naciones Unidas, 1948).y de ella depende la calidad de hombres y mujeres que se obtendrán como producto de la vida familiar. Para llegar a configurar la familia se necesita atravesar por algunos pasos: a) obtener la edad para el matrimonio, b) conocer a la persona idónea, c) noviazgo, d) matrimonio, entendido este como la unión legal entre un hombre y una mujer que adoptan una vida juntos. Por supuesto que se llega al matrimonio después de atravesar por ese periodo de noviazgo que es trascendental para llegar a configurar dicho estado civil.

El matrimonio está presente en la sociedad hondureña, ya que las parejas se casan y forman sus familias, pero también viven en cohabitación y forman sus familias; es decir, la formación de parejas es variada (Quilodran, 2003; Rodríguez, 2004; Arriagada, 2002; Esteve, Lesthaeghe y López-Gay, 2012; López, 2010). La existencia de una dualidad en la formación de parejas ha estado presente y lo seguirá estando en los países latinoamericanos. La cohabitación aumenta mientras el matrimonio disminuye. 


\section{FORMAS DE MATRIMONIO Y COHABITACIÓN EN HONDURAS, SEGÚN FACTORES SOCIODEMOGRÁFICOS}

La relación de pareja, ya sea en matrimonio o cohabitación, puede pensarse como un resultado del comportamiento sociocultural de las personas; aunque también existen otros factores que pueden influir en este hecho. En tal sentido, la encuesta utilizada para la realización del presente trabajo incluye información sobre las características sociodemográficas de los jóvenes entre los 18 y 29 años entrevistados, lo que permitió elaborar la descripción que se presenta a continuación.

\section{Matrimonio y cohabitación por sexo}

La cohabitación y el matrimonio presentan un comportamiento similar entre hombres y mujeres; para el caso, del $100 \%$ de las mujeres que se encuentran en unión, un $75 \%$ se encuentran en cohabitación y $25 \%$ se encuentran casadas legalmente. Para los hombres, el $78 \%$ se encuentra en cohabitación y un $22 \%$ está legalmente casado. Se observa que los hombres cohabitan más que las mujeres, con una diferencia mínima, pero observable; asimismo, son las mujeres las que más se casan y, en consecuencia, son los hombres los que mantienen mayores niveles de cohabitación (ver tabla 1).

Tabla 1. Estado civil de hombres y mujeres jóvenes de 18 a 29 años.

\begin{tabular}{|l|c|c|}
\multicolumn{1}{|c|}{ Tipo de unión } & Mujeres \% & Hombres \% \\
\hline Casado & 24.7 & 21.5 \\
\hline Cohabitando & 75.3 & 78.5 \\
\hline Total & 100 & 100 \\
\hline
\end{tabular}

Fuente: elaboración propia con datos de ENDESA 2011- 2012.

La cohabitación ha coexistido en la población hondureña desde tiempos de la Colonia y en el transcurso del proceso histórico se ha mantenido por sobre el matrimonio y la unión de hecho (Sánchez, 2013).

Matrimonio y cohabitación por área de residencia

La cohabitación es un fenómeno que se da en todos los departamentos del país, pero 
en cada uno de ellos se presenta con diferente intensidad, de igual manera hay diferencias según zona de residencia.

En el país en los últimos años, según la ENDESA 2012, más del $50 \%$ de la población es urbana y la tendencia es hacia el crecimiento de la población de las zonas urbanas, esto se debe al proceso de industrialización, puesto que permite el acceso al mercado laboral de hombres y mujeres. La cohabitación es mayor en las zonas rurales en comparación con las zonas urbanas. En el caso de los hombres es más notoria la diferencia entre zonas de residencia, ya que para la urbana la cohabitación es del30\% y para la rural es del $49 \%$.

La población de Honduras para 2011- 2012, refleja una mayor prevalencia de la cohabitación en las zonas rurales, para hombres y mujeres, del $49 \%$ y $40 \%$, respectivamente. En conclusión, quienes más cohabitan son los hombres (ver gráfico 1).

Gráfico 1. Tipo de unión de hombres y mujeres en edades de 18 a 29 años, según zona de residencia

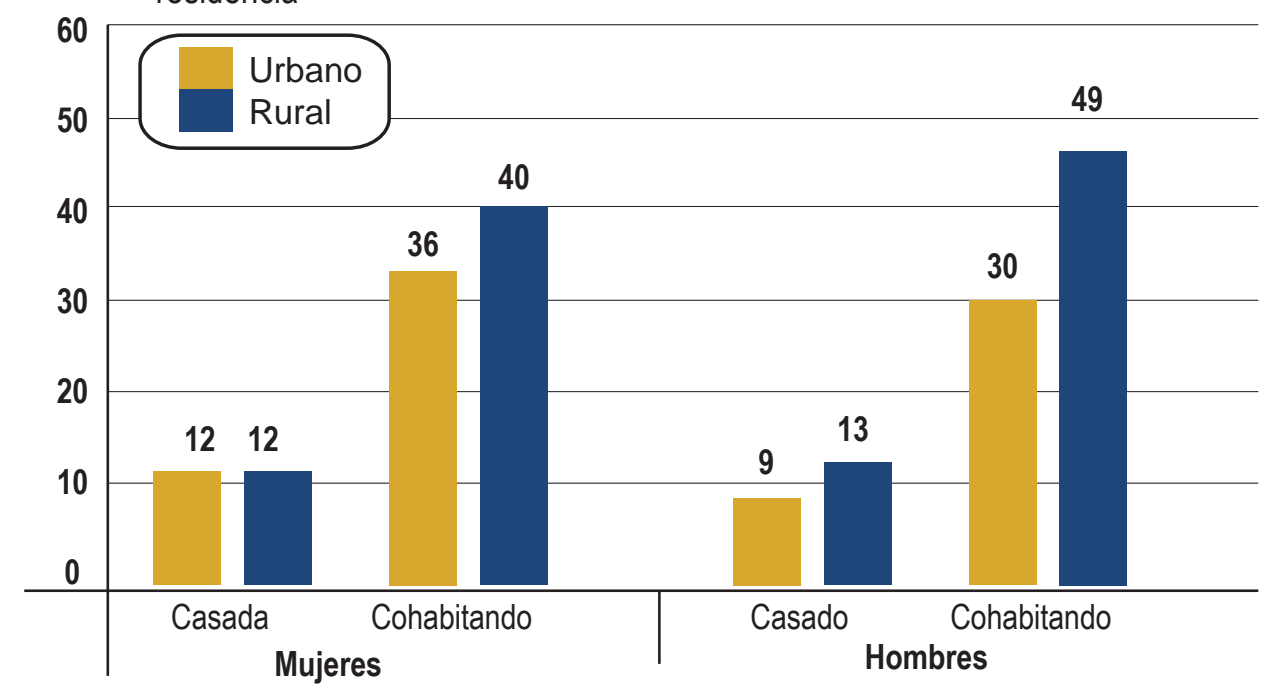

Fuente: elaboración propia con datos de ENDESA 2011- 2012.

La zona de residencia tiene influencia en la decisión que toman las personas para estar en matrimonio o en cohabitación; Honduras tiene una mayor prevalencia de la cohabitación en las zonas rurales. 


\section{Matrimonio y cohabitación según grupos de edad}

La cohabitación es un fenómeno que cambia según la variable que se está observando de acuerdo a la edad de los encuestados; en las mujeres que deciden unirse ya sea en matrimonio o en cohabitación, a la edad de los 18 años, predomina la relación de pareja en unión libre, siendo más bajo el porcentaje de mujeres que la inician con el matrimonio legal $0.31 \%$ a los 18 años y $0.81 \%$ a los 19 .

Ambas formas de unión aumentan en el transcurso del ciclo de vida de las mujeres, pero es la cohabitación la que tiene los más altos porcentajes; las mujeres que se unen a los 18 años deciden hacerlo en su mayoría conviviendo en un $5.45 \%$ y las que se casan tienen un $0.31 \%$ del total general de todas las edades de 18 a 29 años. Las mujeres mantienen las tasas de cohabitación hasta la edad de 19 años, que es donde empieza a descender y el matrimonio empieza a aumentar. El matrimonio en el caso de las mujeres aumenta considerablemente a partir de los 24 años y la cohabitación se mantiene a la edad de 24 años por sobre el matrimonio (ver gráfico 2).

Los hombres que deciden unirse en pareja a la edad de 18 años, de igual forma que las mujeres, en mayor porcentaje los hacen conviviendo sin matrimonio y va aumentando en el transcurso de las edades del $2.24 \%$ a los 18 años al $7.52 \%$ a los 23; alcanzando un $10 \%$ a los 27 años; al igual que las mujeres, empieza un descenso de la cohabitación a los 29 años. Pero, a diferencia de las mujeres, el matrimonio no aumenta a la edad de 29 años, sino que disminuye del $4,4 \%$ a los 28 años al 3,6\% a los 29 (ver gráfico 2).

El tipo de unión que predomina con relación a la edad en todas las edades seleccionadas para la investigación, de acuerdo a los objetivos planteados es la cohabitación. Al respecto, no todas las personas después de cohabitar deciden casarse, pues siguen manteniendo relaciones de cohabitación. Pero, al llegar a edades más adultas toman decisiones que se ven influenciadas por el avance de la edad y factores de convivencia. Si la experiencia en la cohabitación no es como lo esperaba, no avanza hacia el matrimonio. Lo que las personas hacen es retrasar su edad al matrimonio, pero no renuncian a él.

Actualmente Honduras está atravesando por la primera transición demográfica. Entonces, como lo explica Rodríguez (2004), se trata de una cohabitación de tradición o modernidad. En el caso de Honduras, una cohabitación de tradición con características modernas pudiera ser una característica para una segunda transición 
demográfica prematura, pero para confirmarlo se tendrá que hacer en una investigación al respecto.

Gráfico 2. Porcentaje de hombres y mujeres que cohabitan según edad

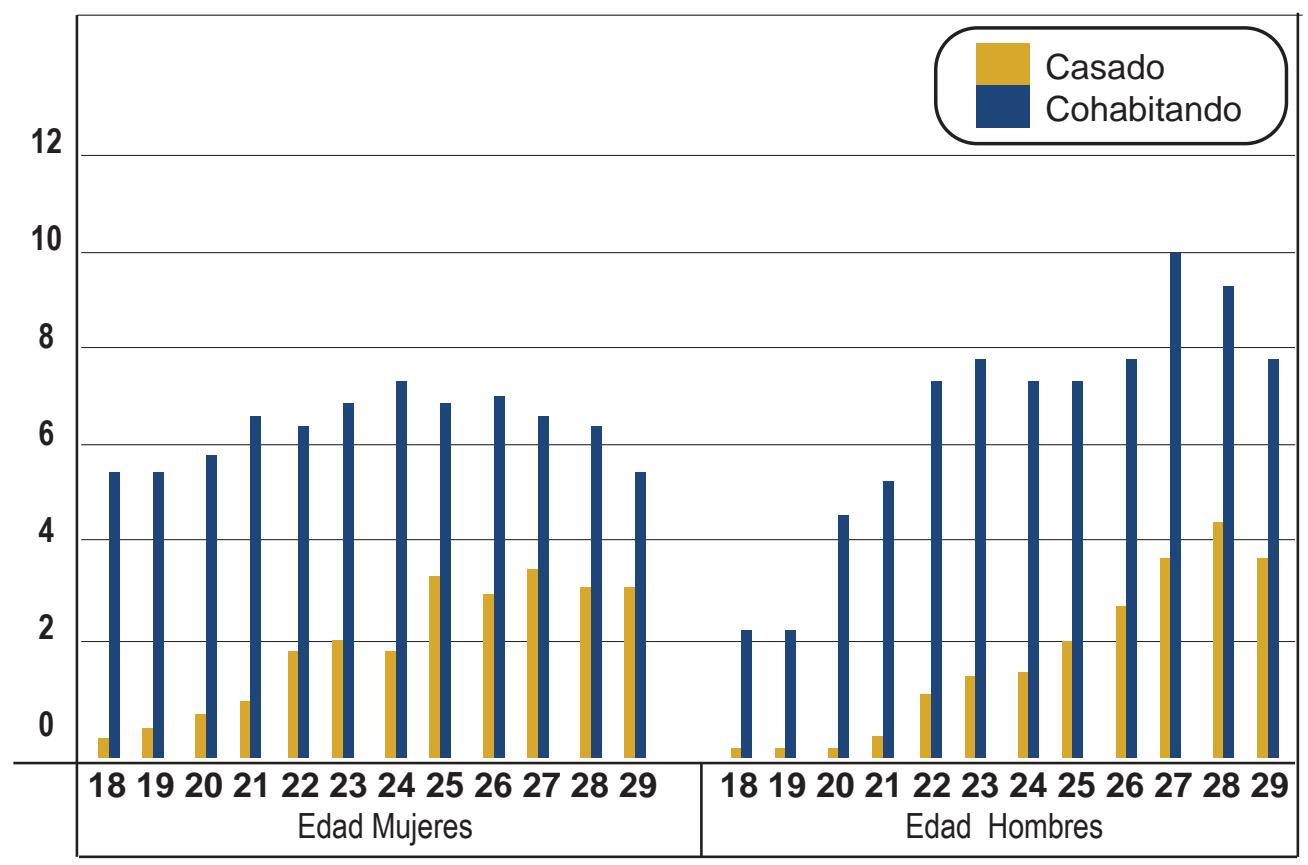

Fuente: elaboración propia con datos de ENDESA 2011- 2012.

Matrimonio y cohabitación por nivel educativo

La variable educación tiene influencia en la decisión que toman las parejas sobre cohabitar o unirse mediante matrimonio. Toda persona que estudia en su curso de vida va avanzando en cuanto a su nivel académico y va planteándose objetivos de vida. Así, hombres y mujeres deciden unirse sin firmar un documento, pero no renuncian al matrimonio, sino que hacen una pausa para alcanzar sus metas y mejorar su nivel académico, postergando de esta manera el matrimonio.

De las mujeres que cohabitan, un 56\% tiene educación primaria, un 38\% educación secundaria, un $3 \%$ no tiene educación y un 3\% educación superior. Para los hombres, un $67 \%$ tiene educación primaria, un $26 \%$ educación secundaria, un $4 \%$ sin educación y un 3\% educación superior (ver gráfico 3). 
El nivel educativo que prevalece en las personas que cohabitan es la educación primaria y en segundo lugar la educación secundaria. De igual forma para las personas que están casadas, la educación primaria es la que presenta porcentajes más altos, seguida de la educación secundaria y en tercer lugar la educación superior.

Gráfico 3. Porcentaje de hombres y mujeres en edades de 18 a 29 años que cohabitan, según nivel educativo

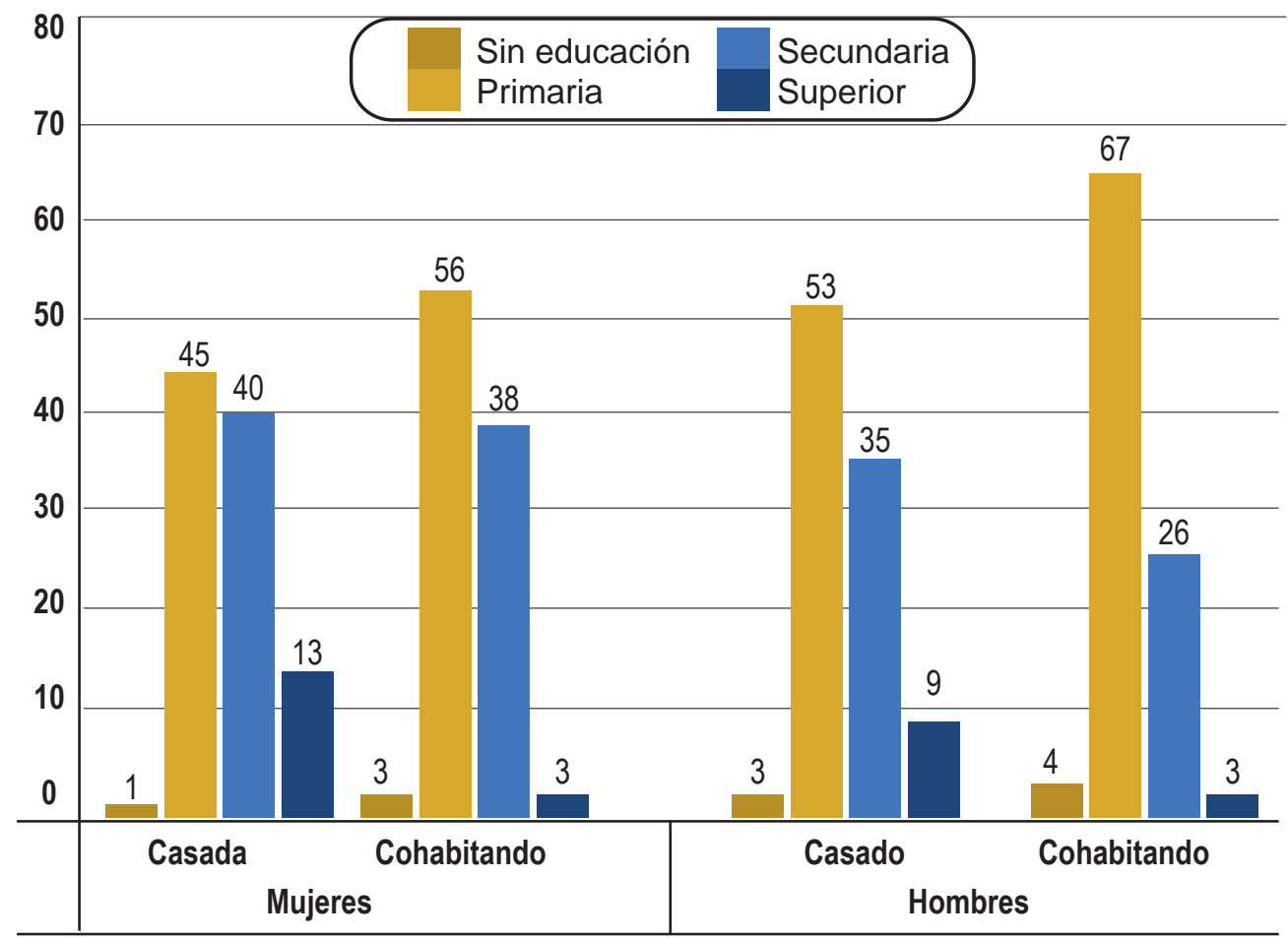

Fuente: elaboración propia con datos de ENDESA 2011- 2012.

El factor educación puede incidir en la decisión de cohabitar o la unión matrimonial, generalmente parejas con nivel educativo de educación primaria y secundaria viven más en cohabitación que en matrimonio. En el caso de las parejas que tienen educación superior, se refleja que tanto en hombre como en mujeres hay ligeros porcentajes más elevados que los de la cohabitación; en otras palabras, las parejas con educación superior se orientan más al matrimonio que a la cohabitación. 


\section{Matrimonio y cohabitación según religión}

La religión tiene influencia en cuanto a la decisión que toman hombres y mujeres para casarse o cohabitar. En el caso de Honduras, para las mujeres en edades de 18 a 29 años de edad se observa que las que no tienen una religión el $89.54 \%$ están en cohabitación, las mujeres católicas están en cohabitación en un $78.40 \%$, las evangélicas protestantes en un $68.82 \%$ y las que tienen otra religión conviven en un $88.06 \%$. Es decir, que independientemente de la religión que tienen las mujeres, la cohabitación es la forma de convivencia que más se practica. También se observa que las mujeres y hombres evangélicos protestantes son los que más se casan legalmente.

El mismo panorama se observa en el caso de los hombres; cualquiera que sea la religión, la cohabitación es más alta en todos los casos, siempre se observa que quienes más se casan son los hombres que pertenecen a la religión evangélica (ver gráfico 4).

Es evidente que la religión protestante influye mucho en el matrimonio, ya que no hacerlo significa que se vive en pecado, lo cual es considerado como adulterio o fornicación; esto le quita a las personas la posibilidad de ejercer cargos o servir en la iglesia y, al mismo tiempo, la posibilidad de estar en plena comunión con Dios.

Gráfico 4. Porcentaje de hombres y mujeres de 18 a 29 años casados y cohabitando, según religión

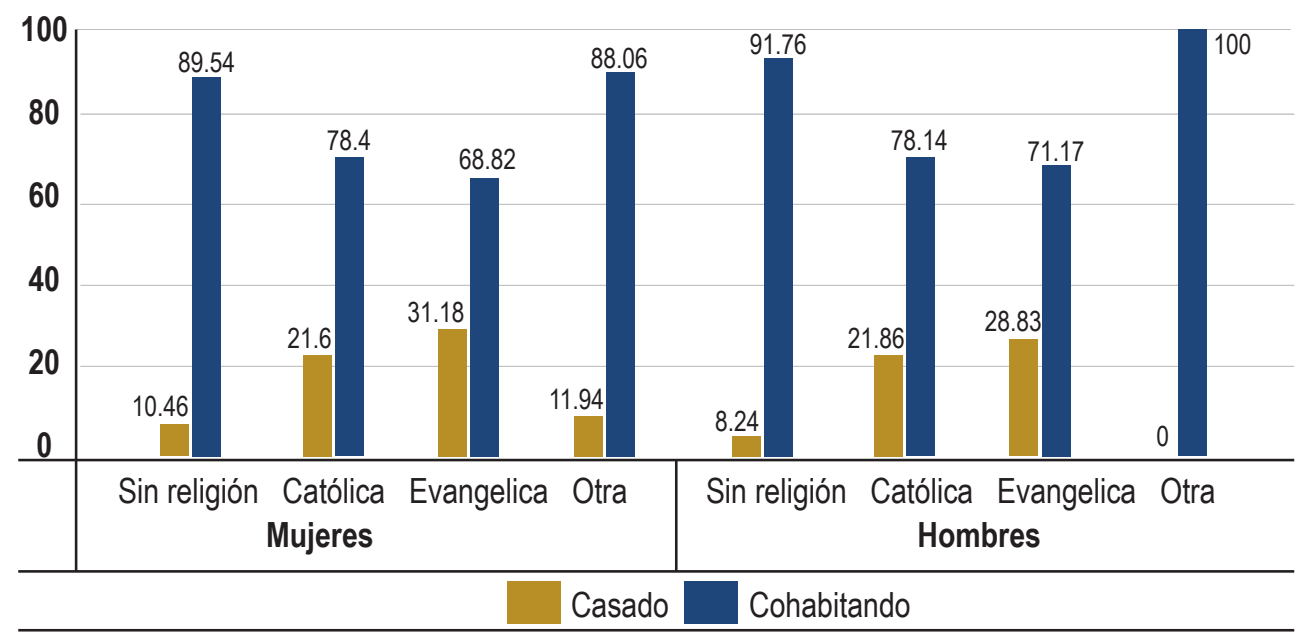

Fuente: elaboración propia con datos de ENDESA 2011- 2012. 
Matrimonio y cohabitación según grupo étnico

Honduras tienen una diversidad de grupos étnicos como garífunas, negro inglés, tolupanes, pech, misquito, nahoa, lenca, tawaka y maya chorti. El grupo predominante son los mestizos.

En todos los grupos étnicos es mayor la cohabitación, se observa un 100\% de cohabitación para los tawaka, en segundo lugar los garífunas y les siguen los misquitos, tolupanes, maya chortí, lencas, negro inglés y nahoa. Las personas que declararon al momento de la aplicación de la encuesta no sabeo ninguna, se consideran los mestizos, tomando en cuenta que dentro de los que declaran que otra pueden encontrarse también mestizos. En el caso de los hombres tolupanes, estos presentan una tendencia hacia el matrimonio, esa situación puede estar condicionada por la cantidad de personas seleccionadas para la muestra, ya que las mujeres declaran una tendencia mayor hacia la cohabitación (ver tabla 2).

Tabla 2. Porcentaje de mujeres y hombres que cohabitan, según grupo étnico

\begin{tabular}{|l|c|c|c|c|c|c|}
\multirow{2}{*}{ Etnia } & \multicolumn{4}{c|}{ Mujer } & \multicolumn{3}{c|}{ Hombre } \\
\cline { 2 - 7 } & Casada & Cohabitando & Total & Casada & Cohabitando & Total \\
\hline Garifuna & 17.8 & 82.2 & 100 & 11.4 & 88.6 & 100 \\
\hline Negro inglés & 43.5 & 56.5 & 100 & 12.5 & 87.5 & 100 \\
\hline Tolupán & 22.6 & 77.4 & 100 & 60.0 & 40.0 & 100 \\
\hline Pech (paya) & 23.5 & 76.5 & 100 & 0.0 & 100.0 & 100 \\
\hline Misquito & 18.6 & 81.4 & 100 & 12.5 & 87.5 & 100 \\
\hline Nahoa & 50.0 & 50.0 & 100 & --- & --- & 100 \\
\hline Lenca & 35.7 & 64.3 & 100 & 30.5 & 69.5 & 100 \\
\hline Tawaka (sumo) & 0.0 & 100 & 100 & 0.0 & 100 & 100 \\
\hline Maya chortí & 23.3 & 76.7 & 100 & 28.9 & 71.1 & 100 \\
\hline Otra & 29.9 & 70.1 & 100 & 27.6 & 72.4 & 100 \\
\hline No sabe/ninguna & 23.9 & 76.1 & 100 & 20.6 & 79.4 & 100 \\
\hline Total & 24.6 & 75.4 & 100 & 21.5 & 78.5 & 100 \\
\hline
\end{tabular}

Fuente: elaboración propia con datos de ENDESA 2011- 2012.

Las raíces culturales están presentes en los procesos que cada individuo atraviesa y ayudan a identificar las diferentes vías de formación de la pareja, en otras palabras, 
la herencia cultural se puede estar produciendo de forma directa a los patrones culturales; es decir, a diferencia de la religión protestante, la cohabitación no tiene una penalización moral entre los grupos étnicos.

Matrimonio y cohabitación según número de hijos promedio

Las mujeres que se encuentran en cohabitación tienen un máximo de 7 hijos, con una media de 2 hijos; en general, la mayoría de mujeres que están casadas o cohabitando presentan una moda de un hijo (ver tabla 3). La cohabitación deja ver que las parejas que se encuentran en ese tipo de unión presentan un mayor porcentaje, siendo las cohabitantes quienes llegan a tener una mayor cantidad de hijos, con relación a las casadas.

Los hombres que se encuentran en cohabitación tienen un máximo de 5 hijos, en este caso se observa una diferencia de dos hijos al compararlo con las mujeres. Esta diferencia puede estar relacionada con el número de parejas que ha tenido la mujer o el hombre, ya que pueden tener hijos de diferentes parejas. El número de hijos promedio de un hombre cohabitante es uno, siendo la moda un hijo, a diferencia de los hombres casados que tienen una media de 2 hijos y un máximo de seis (ver tabla 3).

Tabla 3. Porcentaje de número de hijos nacidos vivos, según tipo de unión

\begin{tabular}{|c|c|c|c|c|}
\multirow{2}{*}{$\begin{array}{l}\text { Número de hijos } \\
\text { vivos }\end{array}$} & \multicolumn{2}{|c|}{ Mujer } & \multicolumn{2}{c|}{ Hombre } \\
\cline { 2 - 5 } & Casada & Cohabitando & Casada & Cohabitando \\
\hline $\mathbf{0}$ & 15.53 & 12.52 & 14.50 & 18.04 \\
\hline 1 & 35.14 & 40.01 & 36.43 & 45.06 \\
\hline 2 & 28.78 & 28.74 & 31.97 & 23.04 \\
\hline 3 & 14.04 & 13.11 & 11.15 & 9.17 \\
\hline 4 & 5.57 & 4.16 & 4.83 & 3.57 \\
\hline 5 & 0.78 & 1.21 & 0.37 & 1.12 \\
\hline 6 & 0.16 & 0.23 & 0.75 & 0.00 \\
\hline 7 & --- & 0.03 & -- & -- \\
\hline Total & 100 & 100 & 100 & 100 \\
\hline
\end{tabular}

Fuente: elaboración propia con datos de ENDESA 2011- 2012. 
Matrimonio y cohabitación según edad de la primera unión y edad de la primera relación sexual

La edad de la primera relación sexual puede estar vinculada con la decisión que toman las mujeres y hombres de cohabitar a edades tempranas, pues existe una coincidencia entre la primera relación sexual y la primera convivencia.

Según el tipo de unión en la que se encuentran las mujeres, se observa que las que cohabitan tuvieron su primera relación sexual a los 17 años, al igual que su primera convivencia fue a la edad mediana de 17 años. En el caso de las mujeres casadas, estas tuvieron su primera relación sexual a los 19 años y su primera convivencia a la edad mediana de 18 años, un año de diferencia con las mujeres que se encuentran en cohabitación. En el caso de la primera relación sexual son dos años de diferencia.

Los hombres presentan diferencias significativas, ya que según su estado civil la edad de la primera relación sexual para los que cohabitan es de 16 años y la edad mediana de la primera convivencia es de 20, se observa una diferencia de 4 años. Tanto los hombres casados como los que cohabitan tuvieron su primera convivencia después de las mujeres (ver tabla 4).

Tabla 4. Edad de la primera relación sexual y primera convivencia, hombresy mujeres, según tipo de unión, jóvenes de 18 a 29 años

\begin{tabular}{|l|c|c|}
\hline & Casada & Cohabitando \\
\hline Mujer & & \\
\hline Edad mediana de la primera relación sexual & 19 & 17 \\
\hline Edad mediana de la primera convivencia & 18 & 17 \\
\hline Hombre & & \\
\hline Edad mediana de la primera relación sexual & 17 & 16 \\
\hline Edad mediana de la primera convivencia & 20 & 20 \\
\hline
\end{tabular}

Fuente: elaboración propia con datos de ENDESA 2011- 2012.

Aunque la iniciación sexual de los hombres es más temprana, la cohabitación y el matrimonio se retrasan más que en el caso de las mujeres. 


\section{La cohabitación y el matrimonio según factores económicos}

1. Matrimonio y cohabitación según participación en el mercado laboral

El ingreso de las personas al mercado laboral es una variable que autores como Oppenheimer (1988), en su Teoría de la pesquisa conyugal, manifiesta que influye en la decisión que toman hombres y mujeres para unirse, ya sea cohabitando 0 en matrimonio. Tanto hombres como mujeres que trabajan se encontraban en cohabitación con un $74.3 \%$, en tanto que las personas casadas representan el $25.7 \%$ (ver gráfico 5).

Gráfico 5.Porcentaje de población que trabaja, según tipo de unión

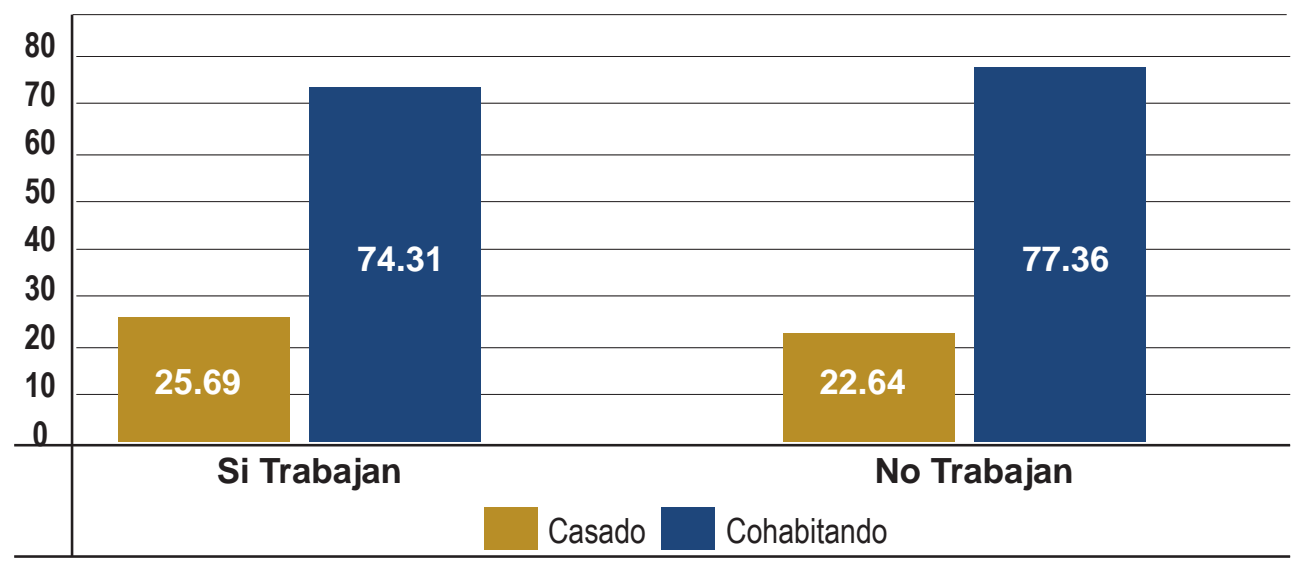

Fuente: elaboración propia con datos de ENDESA 2011- 2012.

Son las parejas que cohabitan las que tienen un mayor porcentaje de representación en el mercado laboral, lo que es congruente con el comportamiento generalizado de la cohabitación entre la población hondureña. En ambos casos, tanto para los que trabaja como para aquellos que no lo hacen, la relación entre cohabitación y matrimonios es de dos a uno.

2. Matrimonio y cohabitación según ocupación

Al analizar los datos de cohabitación y matrimonio según tipo de ocupación, se observan los siguientes resultados: en el caso de las mujeres que cohabitan y trabajan, se desempeñan más en el área de las ventas, los servicios, actividades agrícolas por cuenta propia, trabajos manuales no calificados y profesionales 
técnicos administrativos. Las mujeres casadas se desempeñan en las ventas y como profesionales técnicos administrativos. Las estadísticas reflejan que el 54\% de las mujeres no se encontraba trabajando al momento de la encuesta, lo que deja ver que la inserción en el mercado laboral para las mujeres es más tardía que para los hombres y que muchas de ellas se están desempeñando su rol en las actividades domésticas.

Los hombres que cohabitan se desempeñan más en los trabajos agrícolas por cuenta propia, trabajos manuales no calificados, prestación de servicios, trabajos manuales calificados y en ventas. Los hombres casados se desempeñan en trabajos agrícolas por cuenta propia, profesionales técnicos administrativos y trabajos manuales no calificados. Los trabajos agrícolas sobresalen en el caso de los hombres que cohabitan, lo cual está relacionado con los porcentajes altos de cohabitación que presentan las zonas rurales (ver tabla 5).

Tabla 5. Ocupación agrupada por sexo, según tipo de unión.

\begin{tabular}{|l|c|c|c|c|c|c|}
\multirow{2}{*}{\multicolumn{1}{c|}{ Ocupación }} & \multicolumn{5}{c|}{ Mujer } \\
\cline { 2 - 8 } & Casada & Cohabitando & Total & Casada & Cohabitando & Total \\
\hline No trabajo & 12.17 & 41.82 & 53.99 & 0.08 & 0.08 & 0.16 \\
\hline Profesionales técnicos administrativos & 3.22 & 2.95 & 6.17 & 3.76 & 4.8 & 8.56 \\
\hline Oficinista & 1 & 1.22 & 2.22 & 0.24 & 0.88 & 1.13 \\
\hline Ventas & 3.4 & 9.7 & 13.1 & 1.52 & 4.72 & 6.24 \\
\hline Agrícola-cuenta propia & 1.25 & 4.42 & 5.67 & 9.05 & 35.71 & 44.76 \\
\hline Actividades domésticas & .21 & 2.52 & 2.73 & 0 & 0 & 0 \\
\hline Servicios & 2.02 & 7.82 & 9.84 & 1.68 & 8.33 & 10.01 \\
\hline Manuales calificados & .38 & 1.19 & 1.57 & 2 & 8.17 & 10.17 \\
\hline Manuales no calificados & 1 & 3.71 & 4.71 & 3.2 & 5.77 & 18.97 \\
\hline Total & 24.65 & 75.35 & 100 & 21.54 & 78.46 & 100 \\
\hline
\end{tabular}

Fuente: elaboración propia con datos de ENDESA 2011- 2012.

En el campo laboral existe una diversidad de ocupaciones las cuales desempeñan hombres y mujeres que se encuentran casados o cohabitando. En el caso de las mujeres predominan las actividades relacionadas con el comercio y los servicios. En el caso de los hombres predominan las actividades agrícolas por cuenta propia y los trabajos manuales no calificados. 
Matrimonio y cohabitación según tenencia de bienes

En Honduras existe un déficit de vivienda para las parejas que se encuentran cohabitando o para aquellas que están en matrimonio. Tanto en hombres como en mujeres se observa que la mayoría no tiene una vivienda propia.

En el caso de las mujeres que cohabitan, apenas el $16 \%$ posee vivienda en conjunto con su pareja y un $6 \%$ posee vivienda sola. Para los hombres, el $27.4 \%$ posee vivienda solo y $7 \%$ en conjunto con su pareja. Para los que están casados se observa que, en el caso de las mujeres, el $13 \%$ no posee casa y para los hombres es el $10 \%$; estos últimos reflejan porcentajes altos en la posesión de casa solos y no con su pareja, lo que responde a patrones machistas en alguna medida 0 al hecho de que es él quien tiene acceso a créditos financieros (ver gráfico 6).

Gráfico 6. Porcentaje de hombres y mujeres de 18 a 29 años que poseen casa

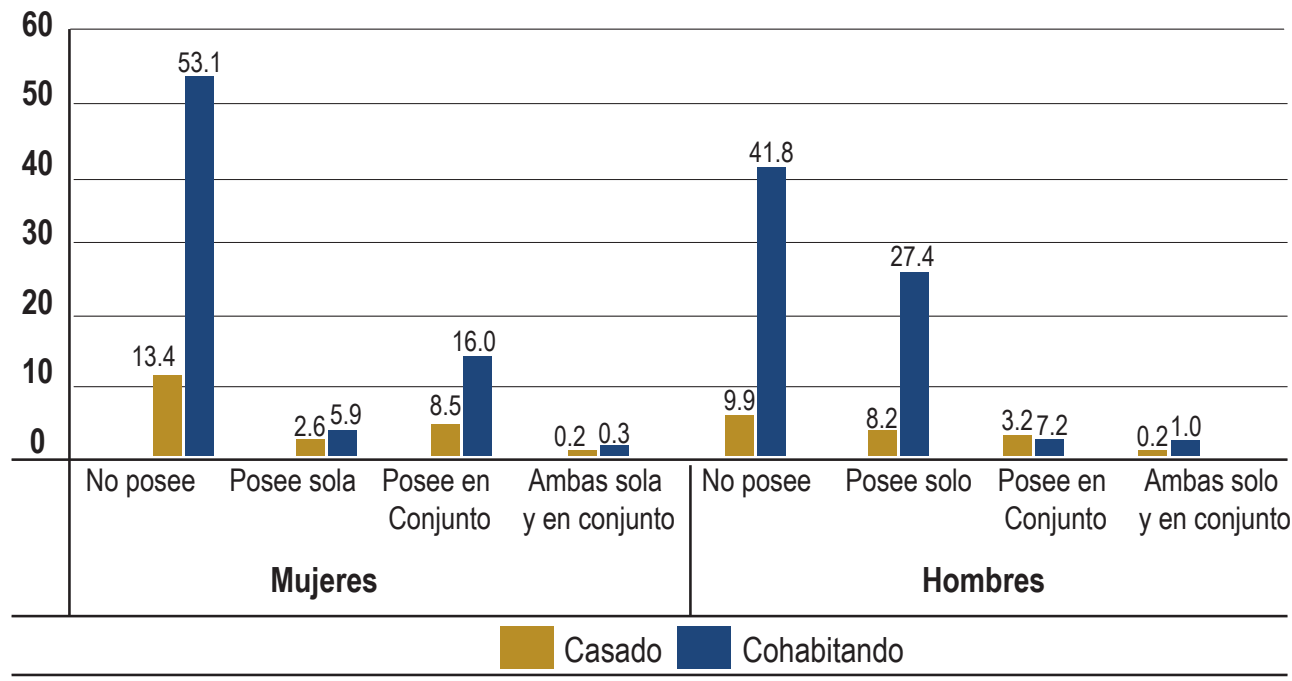

Fuente: elaboración propia con datos de ENDESA 2011- 2012.

En general se observa que la mayoría de las mujeres cohabitando no posee casa, por lo que se intuye que está a nombre de la pareja. En tenencia de terrenos, las mujeres entrevistadas que declaran no poseer terrenos representan el $67 \%$ de las que cohabitan y $21 \%$ de las casadas. Las que tienen terrenos solas representan el $5 \%$ y $2 \%$ para las que cohabitan y están casadas; mientras que las que tienen bienes en conjunto tienen un $3 \%$ más alto que el porcentaje de las casadas, que es del $2 \%$ (ver gráfico 7). 
La mayoría de mujeres cohabitando no posee terrenos. El en caso de los hombres, un porcentaje significativo no posee terreno, es decir, el $53 \%$; el $22 \%$ tiene terrenos solo a su nombre, al igual que en la tenencia de casas. Los hombres encuestados declararon tener más bienes que las mujeres, tanto para los que cohabitan como para los casados: $22 \%$ y $7 \%$, respectivamente. Los porcentajes de tenencia de terrenos en conjunto son similares a las de las mujeres (ver gráfico 7).

Gráfico 7. Porcentaje de hombres y mujeres de 18 a 29 años que poseen terreno

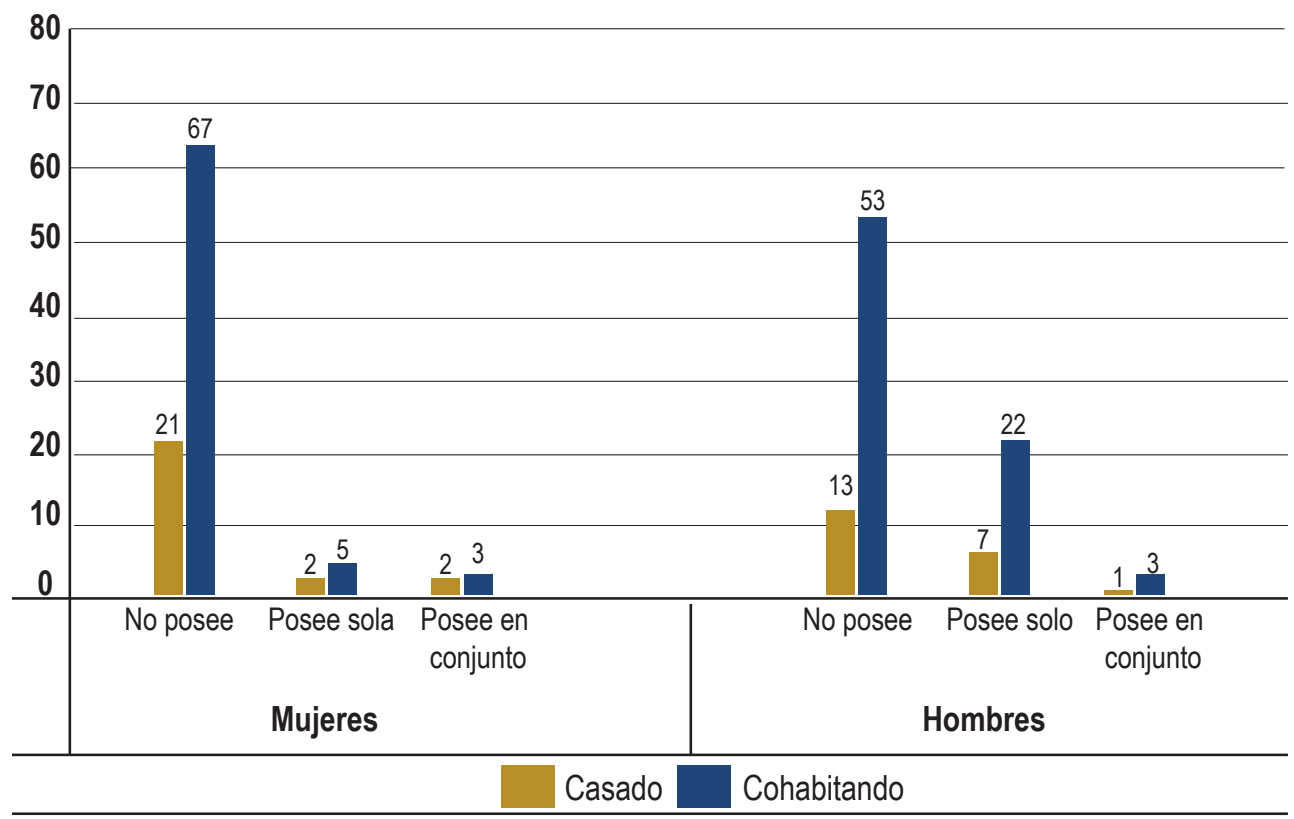

Fuente: elaboración propia con datos de ENDESA 2011- 2012.

En la tenencia de bienes, las casas son los bienes que las personas más poseen en conjunto, independientemente del tipo de unión. La tenencia de terrenos se da más de forma individual y es más representativo para los hombres.

Uno de los beneficios en la tenencia de bienes manifiesta una maximización de la utilidad a favor del conjunto familiar. Las personas que se encuentran en cohabitación tienen más bienes solos que en conjunto, lo que refleja la falta de protección que tiene la pareja al momento una separación. 


\section{MATRIMONIO Y COHABITACIÓN: TENDENCIA DE JÓVENES DE 18 A 29 AÑOS, 2005-2006 Y 2011-2012}

Al comparar los datos del periodo 2005-2006 y 2011- 2012 de la ENDESA, se identifica un incremento en la cohabitación, obedeciendo esto a los patrones ya existentes entre la población, los cambios en las formas de vida de las personas, la inserción de las mujeres en ocupaciones asalariadas dándoles un mayor grado de autonomía y a la búsqueda de mejores oportunidades de empleo y estudio.

La cohabitación en Honduras para el periodo 2005-2006 en jóvenes de 18 a 29 años presenta una tendencia positiva, es decir, en aumento, ya que pasa del $68.1 \%$ al $76 \%$. En cambio, el matrimonio va en declive, pues pasa del 31.9\% al 24\% en 2012 (ver gráfico 8).

Gráfico 8. Tendencia de la cohabitación y el matrimonio 2005-2012, jóvenes de 18 a 29 años

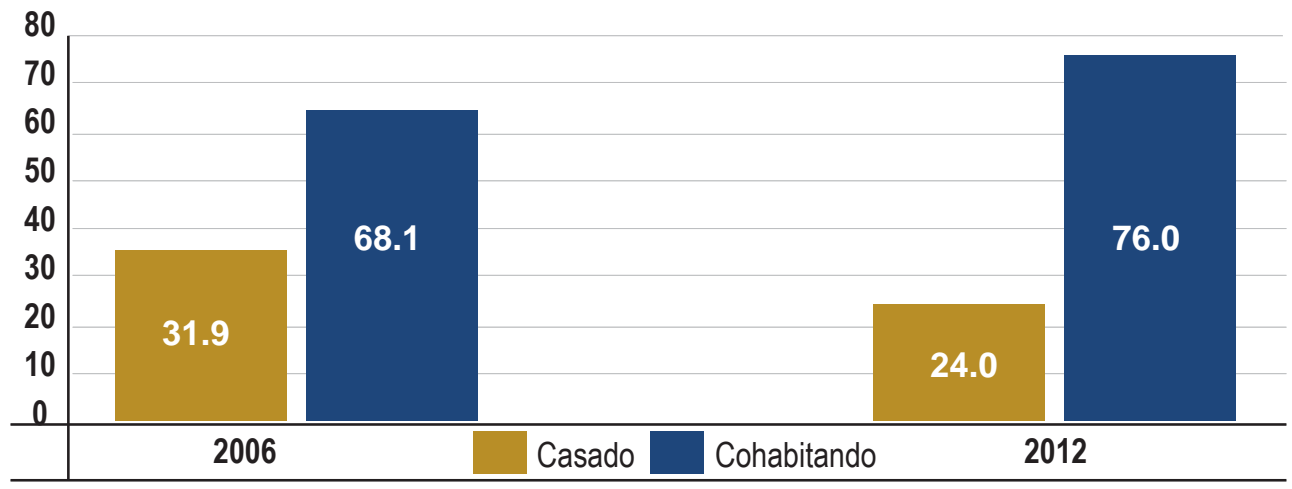

Fuente: elaboración propia con datos de ENDESA 2011- 2012.

Tendencia de la cohabitación por zona de residencia

Según la zona de residencia, se presenta una tendencia al incremento de la cohabitación. El porcentaje de personas que declararon que cohabitaban en $2005-2006$ en el área rural era del $68.36 \%$ y para el $2011-2012$ fue del $76.98 \%$. De igual forma, la zona urbana presenta un aumento del $67.81 \%$ en 2005-2006 y al 74.76\% en 2011-2012 (ver gráfico 9).

Para las personas que se encuentran casadas según lugar de residencia, en el área 
urbana presentan una disminución, era del $32.19 \%$ para 2005-2006 y para el 2011- 2012 disminuyó al $25.24 \%$; de igual forma, el área rural presenta una disminución en el matrimonio, en 2005-2006 el porcentaje de personas casadas era de $31.64 \%$ y para $2011-2012$ de $23.02 \%$ (ver gráfico 9 ).

Gráfico 9. Tipo de unión según zona de residencia

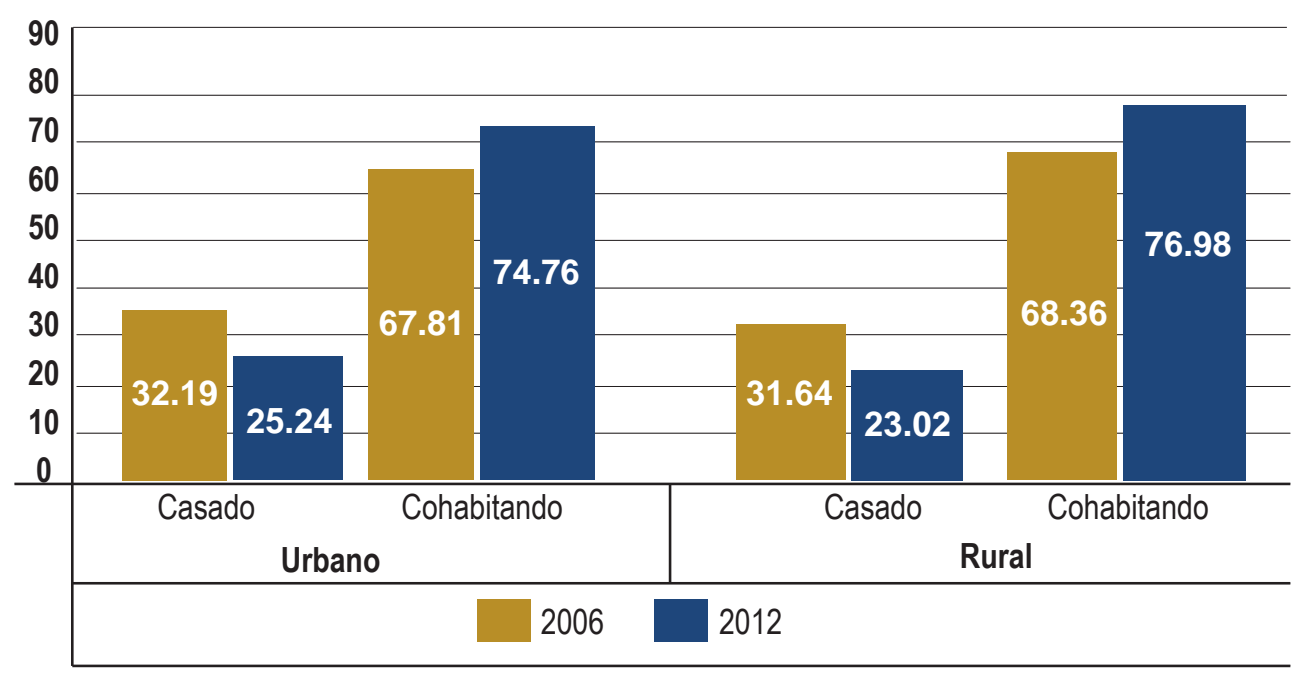

Fuente: elaboración propia con datos de ENDESA 2011- 2012.

\section{MATRIMONIO Y COHABITACIÓN: MODELO DE REGRESIÓN LOGÍSTICA}

De las variables descritas en los apartados anteriores relacionadas con características sociodemográficas, factores socioeconómicos y la razón de encontrarse en unión, ya sea en matrimonio o en cohabitación en un contexto multivariado, se utilizó en el modelo de regresión logística las variables que se muestran más significativas, las cuales incluyen: la religión, el número de uniones, tenencia de casa, número de hijos, zona de residencia, edad de la primera relación sexual, edad de la primera convivencia, nivel educativo y nivel de instrucción.

Dentro de estas variables se identificaron 12 que son consideradas como estadísticamente significativas, con un nivel de confianza del 95\%. De estas, las que presentan un impacto directo son número de hijos, número de uniones, edad de la primera relación sexual, edad de la primera convivencia y nivel de educación secundaria. Las 
variables que se relacionan de manera inversa son: religión, tenencia de casa, residencia urbana, nivel de educación primaria, educación superior y sin instrucción.

La edad de la primera relación sexual y de la primera unión están directamente relacionadas una con la otra, tal como se explica en la teoría. Una enlaza el siguiente paso, después de la primera relación sexual las mujeres deciden estar en unión. Si las personas tienen su primera relación sexual a edades jóvenes, la probabilidad de establecer una unión a esa edad aumenta. El análisis que se hace del modelo va enfocado a las mujeres que se encontraban en unión al momento de la entrevista (ver tabla 6).

Tabla 6. Modelo de regresión logística, factores que inciden en la cohabitación y el matrimonio

\begin{tabular}{|c|c|c|c|c|c|c|}
\hline cohab1829 & Coef. & $\begin{array}{l}\text { Robust } \\
\text { Std. Err. }\end{array}$ & $\mathbf{z}$ & $p>|z|$ & \multicolumn{2}{|c|}{ [95\% Conf. Interval] } \\
\hline rel_catol & -199813 & 0627803 & -3.18 & 0.001 & -32.28602 & -0767658 \\
\hline rel_evang & -1190381 & 0622901 & -1.91 & 0.056 & -2411244 & 0030481 \\
\hline uniones_una & -4459389 & 0479315 & 9.30 & 0.000 & -3519947 & 5398828 \\
\hline tenen_casa & 2287659 & 0382735 & -12.16 & 0.000 & 5403683 & 3903388 \\
\hline num_hijos & -5572245 & 615242 & 37.18 & 0.000 & 2.167074 & 2408244 \\
\hline resi_urba & -2939823 & 0480335 & -13.80 & 0.000 & 1998384 & 4780915 \\
\hline edad_convi & -7716046 & 053301 & 6.12 & 0.000 & 6671366 & 3881261 \\
\hline nivel_pricom & -1510675 & 0426194 & 14.48 & 0.000 & -2345999 & 8760727 \\
\hline nivel_seccom & -3083814 & 0624075 & -3.54 & 0.000 & 1860649 & 067535 \\
\hline educ_sup & -102836 & 0900737 & -1.16 & 0.245 & 1860649 & 4306979 \\
\hline nivel_sinintr & -4076414 & 0980603 & -4.16 & 0.000 & -5998361 & 0737052 \\
\hline _cons & -3106773 & 105383 & 29.48 & 0.000 & -331332 & 2154467 \\
\hline
\end{tabular}

Fuente: elaboración propia con datos de ENDESA 2011- 2012. 


\section{DISCUSIÓN Y CONCLUSIONES}

Honduras es un país en el cual la cohabitación ha existido junto al matrimonio, la primera siempre ha sido mayor y en la actualidad se presenta la tendencia al incremento, lo que disminuye los casos de matrimonio en el país. Las personas están retrasando en algún momento su edad para llegar al matrimonio, pero no están renunciado a él; sin embargo, hay mayor inclinación a la cohabitación. La zona de residencia marca diferencia en el comportamiento de los datos, la más significativa es el predominio de la cohabitación en promedio de hombres y mujeres en el área rural.

Existen diferencias notorias en cuanto a la edad y la cohabitación o matrimonio; pues la cohabitación es alta desde edades tempranas, para el caso desde los 18 años y menos, manteniéndose la cohabitación en niveles estables hasta las edades adultas con tendencia al aumento; lo que ocurre con el matrimonio es que este aumenta a medida que va avanzando la edad. Esto significa que mientras unas personas se casan, otras personas entran a formar parte de la cohabitación, haciendo que se mantengan los porcentajes. Los jóvenes entre los 18 y 29 años se casan más a edades adultas, pero cohabitan en edades jóvenes y media adulta.

En su mayoría, los jóvenes entre 18 y 29 años que cohabitan cuentan con un nivel educativo de educación primaria y secundaria. Los jóvenes entre 18 y 29 años con educación superior tienden a optar por el matrimonio. Las diferencias que se presentan son bien marcadas.

Se partió de la premisa de que la religión que tiene influencia directa para que las personas se casen, sin embargo, esto no se ve reflejado en los datos, ya que la cohabitación es mayor independientemente de la religión de las personas, siendo las católicas quienes más cohabitan y solo en el caso de las parejas que practican la religión protestante se observaron menores niveles de convivencia a favor del matrimonio.

Las mujeres mantienen la pauta de coincidencia en la edad de la primera relación sexual con la edad de la primera convivencia, mientas que los hombres tienen su primera relación sexual a los 16 años y según, su declaración en la encuesta, su primera unión es a los 20 años. Al respecto, se presenta una diferencia importante con relación a las mujeres. Las que se encuentran cohabitando empiezan su actividad sexual y su primera convivencia a más temprana edad en relación a las casadas. 
La misma situación se da en los hombres, con la diferencia de que la convivencia de los casados y los cohabitantes la presentan a los 20 años, tomando en cuenta que es la edad mediana.

Los hombres que cohabitan se desempeñan más en ocupaciones como la agricultura por cuenta propia, trabajos manuales no calificados, servicios manuales calificados; las mujeres en ventas, servicios agrícolas por cuenta propia, trabajos manuales no calificados.

Las casas son los bienes que más se poseen en conjunto, independientemente del tipo de unión. Los terrenos se tienen más de forma individual. Quienes más bienes en conjunto poseen son las personas que se encuentran en cohabitación; pero de la existencia de bienes, la forma de posesión predominante es la individual para el caso de los hombres.

En Honduras, la tendencia de la cohabitación de jóvenes entre 18 y 29 años entre el periodo 2005-2006 y 2011- 2012 va en aumento; sin embargo, el matrimonio presenta una disminución. Según la zona de residencia, en la zona urbana y en la rural se presenta un aumento en la cohabitación; contrario al matrimonio, ya que este disminuyó.

Las parejas de jóvenes entre 18 y 29 años forman sus familias, ya sea casándose 0 cohabitando, pero las pautas de formación de familia se siguen manteniendo a favor de la cohabitación, la cual no disminuye, sino que aumenta. La cohabitación es un fenómeno en el cual la mujer está en condición de cohabitación de igual forma que el hombre, pero quienes están más a favor de una cohabitación son los hombres con relación a las responsabilidades que esto trae consigo.

En los niveles educativos más bajos es donde se presenta con mayor intensión la cohabitación. La edad de la primera relación sexual tiene influencia en la decisión que toman hombres y mujeres al momento de estar en unión, teniendo esta una mayor influencia en las mujeres y más aún en las mujeres que se casan.

Los factores que presentan mayor significancia estadística que influyen en la unión, según el modelo de regresión lineal estimado, son: cuando el número de hijos es menor o igual a 3, edad de la primera relación sexual, edad de la primera convivencia y el nivel de educación secundaria. De manera inversa a la unión, el modelo identificó: a la religión católica o evangélica, tenencia de casa, residencia urbana, nivel de educación primaria y educación superior. 


\section{AGRADECIMIENTOS}

A la Dirección de Investigación Científica y Posgrado de la UNAH por darme la oportunidad de publicar parte de mi trabajo de tesis. A la coordinadora de la Maestría en Demografía y Desarrollo, doctora Marysabel Zelaya y al máster Manuel Flores, por sus contribuciones al tema de esta investigación y, finalmente, a la máster María Auxiliadora López M., quien ha guiado y orientado el presente trabajo.

\section{BIBLIOGRAFÍA}

Arriagada, I. (2002). Cambios y desigualdad en las familias latinoamericanas. Revista de la CEPAL, 77.Recuperado de: http://www.eclac.cl/publicaciones/xml/9/19349 /lcg2180e_arriagada.pdf

Esteve, A.; Lesthaeghe, R. and López-Gay, A. (2012). The Latin American Cohabitation Boom, 1970-2007. Population and Development Review, 38, 55-81. Doi: 10.1111/j.1728-4457.2012.00472.x

Congreso Nacional de la República de Honduras. (1984).Código de la Familia. Decreto 76-84. Recuperado de: http://www.poderjudicial.gob.hn/juris/Leyes/Codigo\% 20de\%20Familia\%20\%28actualizada-07\%29.pdf

Grace, K. and Sweeney, S. (2014). Pathways to marriage and cohabitation in Central America.Demographic Research, 30, 187-226.

Kalmijin, M. (2011). The influence of mens in come an employment on marriage and cohabitation testing Oppenheimer s Theory in Europe. European Journal of Opupation, 269-293. Doi: 10.1007/s10680-011-9238-x

López, L. (2010).Uniones conyugales y distancia social en América Latina. Tesis doctoral. Centro de Estudios Demográficos, Universidad Autónoma de Barcelona. Recuperado de: http://tdx.cesca.cat/bitstream/handle/10803/32085/lalr1de 1.pdf?sequence $=1$

Lesthaeghe, R. (2012). Videoconferencia sobre el boom de la cohabitación en América Latina.Madrid: Fundación BBVA.

Lesthaeghe, R. (1994). Una interpretación sobre la segunda transición demográfica en los países occidentales. Demografía y políticas públicas. Gasteiz Victoria: EMAKUNDE, Instituto Vasco de la Mujer.

Quilodrán, J. (2003). La familia, referentes en transición. Papeles de Población, 37(9). Recuperado de: http://www.redalyc.org/articulo.oa?id=11203703 
Rodríguez, J. A. (2004). Cohabitación en América Latina: ¿Modernidad, exclusión o diversidad? Papeles de Población, 10(40), 97-145. Recuperado de:http://www.re dalyc.org/articulo. oa? id $=11204008$

Sánchez, R. (2013).El desarrollo de las políticas reformistas liberales de Marco A. Soto y su influencia en la estructura de la población 1876-1887. Tesis de maestría. Universidad Nacional Autónoma de Honduras.

Secretaría de Salud de Honduras, Instituto Nacional de Estadística e ICF International. (2013). Encuesta nacional de salud y demografía 2011-2012. Tegucigalpa.

Secretaria de Salud de Honduras, Instituto Nacional de Estadísticas (INE) Y Macro Internacional. (2006). Encuesta nacional de salud y demografía 2005 - 2006. Tegucigalpa.

Von de Kaa, D. (2002). The idea of secon demographic transition in industrialized countries. Paper presented at the sixth Welfare Policy Seminar of the National institute of Population and social Security. Tokyo, Japón. Recuperado de: www.ipss.go.jp/webj-ad/webjournal.files/population/2003_4/kaa.pdf 